\title{
Rasputin, more promiscuous than ever: a review of G3BP
}

\author{
KATHARINE IRVINE ${ }^{1}$, RENEE STIRLING ${ }^{2}$ DAVID HUME ${ }^{1}$ and DEREK KENNEDY*,2 \\ ${ }^{1}$ Institute for Molecular Bioscience, University of Queensland, St Lucia, Australia and ${ }^{2}$ Eskitis Institute for Cell and Molecular Therapies, \\ Griffith University, Nathan, Australia
}

\section{CONTENTS}

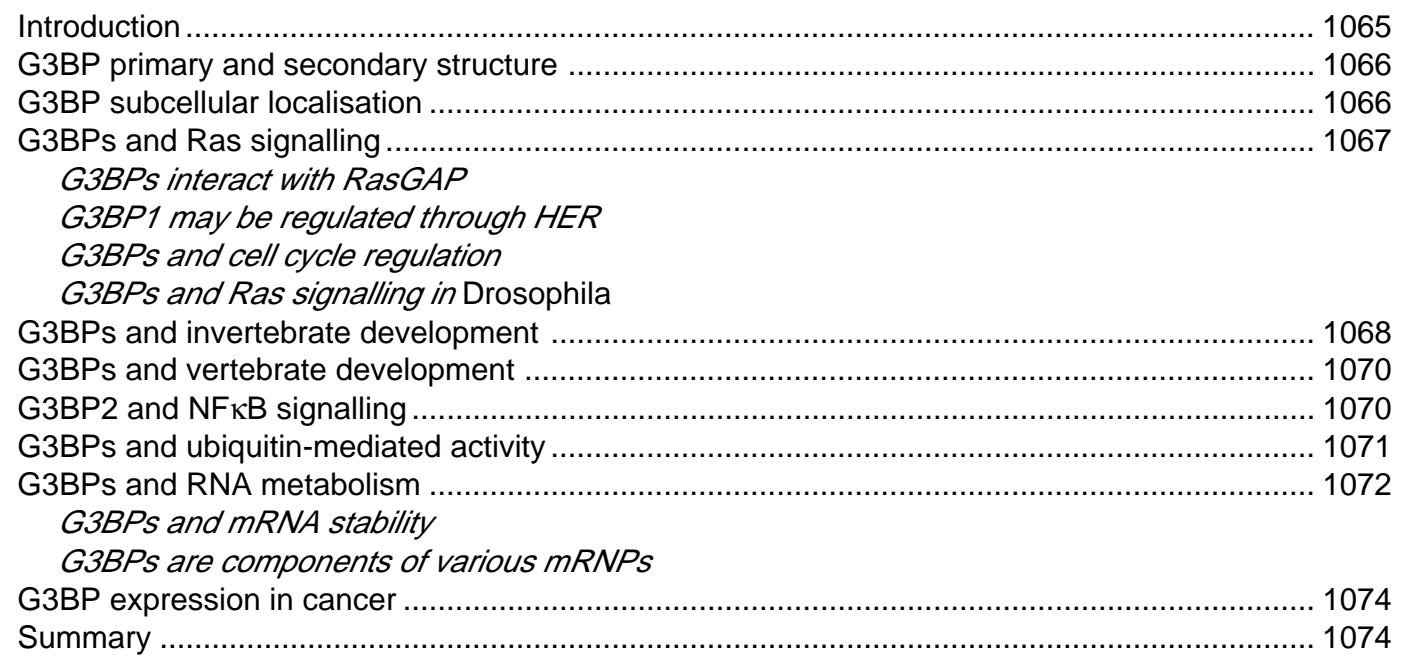

KEY WORDS: G3BP, Rasputin, Bre5, RNA-binding protein

\section{Introduction}

In the year 2000, the Drosophila homologue of Ras-GTPase activating protein $\mathrm{SH} \underline{3}$ domain binding protein (G3BP) was reported and named Rasputin because of its genetic interactions with members of the Ras signalling pathway. G3BP and its mysterious nineteenth century Russian namesake both resided in positions of great power, one in the imperial court and the other at the nexus of signal transduction; and in both cases their role in these positions is disputed. This review endeavours to consolidate the current, somewhat disjointed literature, so as to create a more cohesive picture of G3BPs and their putative functions.

The G3BP family of proteins has been the subject of a small, but extraordinarily diverse literature since G3BP1 was first isolated in 1996 (Parker et al., 1996). The family is evolutionarily conversed throughout eukaryota. Mammals boast at least three G3BPs:
G3BP1, 2a and 2b which are the products of two distinct genes (Kennedy et al., 2001). The proteins are relatively ubiquitously expressed and seem to play important roles in several biologies, yet their actual functions remain enigmatic. Nor is it clear whether the highly homologous family members play distinct, overlapping or complementary roles in the cell. This may be attributed to the fact that research in the field has taken an extremely non-processive

\footnotetext{
Abbreviations used in this paper: $\mathrm{CE}$, convergence and extension; $\mathrm{CRD}$, coding region instability determinant; CRS, cytoplasmic retention sequence; CSF, colony stimulating factor; EGF, epidermal growth factor; G3BP, GTPase activating protein SH3 domain binding protein; GAP, GTPase activating protein; HER, human epidermal growth factor receptor; mRNP, messenger ribonucleoprotein particle; NTF, nuclear transport factor; PKC, protein kinase C; PVR, proliferative vitreoretinopathy; RHD, Rel homology domain; RNP, ribonucleoprotein particle; RPE, retinal pigment epithelium; RRM, RNA recognition motif; TIA, T-cell internal antigen; USP, ubiquitin-specific proteases.
}

*Address correspondence to: Dr. Derek Kennedy. School of Biomolecular and Biomedical Sciences, Griffith University, Nathan 4111, Australia. Fax: +61-7-3875-7276. email: derek.kennedy@griffith.edu.au 
path, in which diverse systems have been used to produce a variety of results (see Table 1). This would seem to indicate that G3BPs are proteins of many and possibly unrelated, functions, which may or may not be cell type specific.

\section{G3BP primary and secondary structure ${ }^{1}$}

G3BP 1 and 2 are encoded by distinct genes on human chromosomes 5 and 4 and mouse chromosomes 11 and 5 respectively. G3BP2b is a splice isoform of G3BP2a, lacking 33 amino acids in the central region (Kennedy et al., 2001). Figure 1 is a linear representation of the three G3BPs showing the arrangement of known domains possessed by these proteins. There is $65 \%$ identity and 74\% similarity between G3BP1 and G3BP2a protein sequences across the mouse and human species.

G3BP C-termini comprise two motifs traditionally associated with RNA binding. These are a canonical RNA Recognition Motif (RRM) and a loosely conserved RGG (arginine-glycine rich) box (Birney et al., 1993). The RRM-containing family of proteins is the largest family of RNA-binding proteins in mammals. The domain consists of two short, loosely conserved motifs, RNP1 and RNP2, separated by an amino acid sequence of variable composition and length (Kennedy et al., 1997). RGG domains are often found in RNA-binding proteins and may confer cooperative binding to RRM motifs (Burd and Dreyfuss, 1994, Ghisolfi et al., 1992, Mayeda et al., 1994). RGG domains have also been shown to influence nuclear translocation. For example, arginine methylation in the RGG box of hnRNPA2 promotes its nuclear localisation (Nichols et al., 2000).

The most highly conserved domain, both between species and within the mammalian G3BP family members, is the N-terminal Nuclear Transport Factor 2-like (NTF2-like) domain (Fig. 1). NTF2 is a small protein involved in RanGTP-dependent nuclear import of proteins through the nuclear pore complex (Ribbeck et al., 1998). Using 3D homology modelling as described elsewhere (Kennedy et al., 1996, Kennedy et al., 1997), we generated an in silica structure of the N-terminal NTF2-like domain of G3BPs. As would be expected from the sequence homology, the motif was shown to assume a similar fold to that of NTF2 (unpublished data). It is therefore possible that some of the functional features of NTF2 might also be conserved. In particular, the presence of this domain raises the possibility that G3BPs might play a role in nuclear transport and, like NTF2, G3BPs may also bind Ran or other small GTPases. Indeed, G3BP1 recently appeared in a list of Ranbinding proteins, although an interaction was not directly shown in that publication (Macara, 2001).

G3BPs' central regions comprise varying numbers of proline rich (PxxP) motifs and an acid-rich domain (Fig. 1). PxxP motifs represent the minimal consensus sequence for protein binding to the conserved aromatic amino acid residues that compose $\mathrm{SH} 3$ domains (Booker et al., 1993, Saksela et al., 1995). SH3 domains are widely distributed signalling adaptor modules. Although G3BP1 was initially isolated as a RasGAP SH3 domain binding protein and all three G3BPs have subsequently been shown to bind the $\mathrm{SH} 3$ domain of RasGAP (Kennedy et al., 2001, Parker et al., 1996),

Note 1: "G3BP" will be used generically throughout this work, like "G3BPs", to describe certain features of the protein family which do not necessitate distinction between the genes.
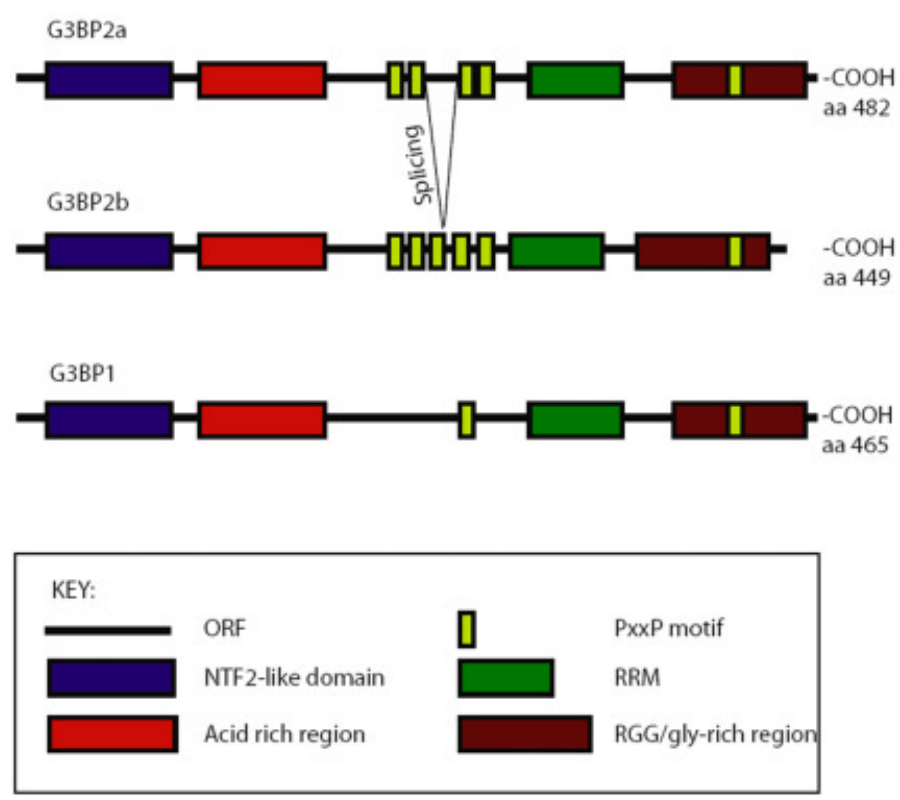

Fig. 1. Comparison of the predicted domain structure of human G3BP2a, $\mathbf{2 b}$ and $\mathbf{1}$. The position of the alternate splicing event which removes 33 amino acids from G3BP2a to generate G3BP2b, thereby creating an additional PXXP motif in G3BP2b is indicated.

PxxP motifs have not been shown to mediate this interaction. In vitro binding assays with G3BP1 and G3BP2 suggested that the NTF2-like domain of these proteins was responsible for RasGAP binding (Kennedy etal., 2001). It is rare, but not unprecedented, for non-proline motifs to bind $\mathrm{SH} 3$ domains (Agrawal and Kishan, 2002).

\section{G3BP subcellular localisation}

G3BP1 and G3BP2a have previously been reported to be primarily cytoplasmic proteins (Parker et al., 1996, Prigent et al., 2000), but both proteins may have the capacity to enter the nucleus (Costa et al., 1999, French et al., 2002, Tourriere et al., 2001). Other researchers have reported further localisations for G3BP1 including: by immunofluorescence, stress granules (Tourriere et al., 2003); and, biochemically, the insoluble cytoplasm in proliferating cells (Gallouzi et al., 1998) and the endoplasmic reticulum in Saccharomyces cerevisiae (Cohen et al., 2003a).

Both G3BP1 and G3BP2 isoforms possess an NTF2-like domain (discussed above) which is known to mediate the nuclear localisation of other proteins, including NTF2 itself (Smith et al., 1998). Accordingly, both G3BP1 and G3BP2a have been observed in cell nuclei in several studies. Tourriere and co-authors (Tourriere et al., 2001) reported the nuclear localisation of phosphorylated G3BP1 in quiescent mouse embryonic fibroblasts and, subsequent to its initial identification as a RasGAP binding protein, G3BP1 was isolated from a HeLa nuclear extract as a functional DNA and RNA helicase (Costa et al., 1999). By contrast, Parker and colleagues did not detect G3BP1 in the nucleus of Epidermal Growth Factor (EGF)-transformed fibroblasts at any stage of the cell cycle (Parker et al., 1996). At least one G3BP2 isoform has also been shown to shuttle between the nucleus and the cytoplasm in 
a cell cycle dependent manner. Contrary to G3BP1, we found G3BP2 to be exclusively cytoplasmic in quiescent fibroblasts and to rapidly enter the nucleus upon serum stimulation (French et al., 2002). We also examined G3BP2 expression by immunohistochemistry in a range of breast cancers, finding that it was overexpressed in a vast majority and nuclear localisation was detected in almost half the cancers studied. The antibody employed in both these studies does not differentiate between G3BP2a and G3BP2b (French et al., 2002). The cytoplasmic localisation of a G3BP2a mutant lacking the NTF2-like domain was studied by Prigent and colleagues (Prigent et al., 2000). Whereas full length G3BP2a was distributed throughout the cytoplasm and at the nuclear envelope, the mutant construct did not localise to the nuclear envelope, suggesting the NTF2-like domain is responsible for targeting G3BP2a to the nuclear envelope in HeLa cells. The CRM-1 nuclear export pathway does not appear to be involved in G3BP2a cellular distribution since G3BP2a cytoplasmic localisation in HeLa cells was not affected by Leptomycin B (Prigent et al., 2000).

\section{G3BPs and Ras signalling}

\section{G3BPs interact with RasGAP}

The majority of publications concerning G3BP1 support a function in cell proliferation and/or survival downstream of Ras. The first G3BP family member to be discovered, G3BP1, was isolated in a screen for proteins that bind the $\mathrm{SH} 3$ domain of Ras GTPase Activating Protein (RasGAP) (Parker et al., 1996). Ras is a small
GTPase responsible for transducing signals from a vast array of receptor tyrosine kinases and other receptors. It acts as a molecular switch, cycling between the active, GTP-bound form and the inactive GDP-bound form. Ras is regulated by GTPase Activating Proteins (GAPs), which attenuate signalling and Guanine nucleotide Exchange Factors (GEFs) which activate Ras via GTP transfer. The N-terminal domain of rasGAP harbours an $\mathrm{SH} 3$ domain flanked by two $\mathrm{SH} 2$ (phosphotyrosine binding) domains. Several potential effectors associating with RasGAP via the $\mathrm{SH} 2$ domain have been identified, none of which require the $\mathrm{SH} 3$ domain, but only six proteins that bind to the $\mathrm{SH} 3$ domain are known (Gigoux et al., 2002, Liu et al., 1997, Tocque et al., 1997). Three of these are the G3BPs.

The SH3 domain of RasGAP is essential for transducing certain signals downstream of Ras in a MAPK-independent manner (Tocque et al., 1997). Several studies have shown that the RasGAP SH3 domain is important in oncogenic Ras signalling pathways (Duchesne et al., 1993; Pomerance et al., 1996; Leblanc et al., 1999). The RasGAP SH3 domain is also involved in cytoskeletal reorganization, cell adhesion and the induction of gene expression in a Ras-independent manner (Leblanc et al., 1998, Medema et al., 1992). Several key studies have now suggested that the RasGAP $\mathrm{SH} 3$ domain plays an essential role in signal transduction in a variety of systems and potential effector molecules have been avidly sought.

G3BP1 was co-immunoprecipitated with the RasGAP SH3 domain from fibroblasts over-expressing the EGF Receptor and subsequently shown to bind to full length RasGAP (Parker et al.,

TABLE 1

\section{SUMMARY OF THE BIOLOGY ASSOCIATED WITH G3BPS}

\begin{tabular}{|c|c|c|c|}
\hline Biology & Comments & Organism/cell lines & Relevant publications \\
\hline \multirow[t]{5}{*}{ RNA Metabolism } & Interaction of G3BP1 with $c-m y c$ mRNA & Mammalian cell lines & Gallouzi et al., 1998, Tourriere et al., 2001 \\
\hline & Interaction of G3BP1 with Tau mRNA & P19 cells & Atlas et al., 2004 \\
\hline & G3BP1 activity as RNA and DNA helicases & HeLa cells & Costa et al., 1999 \\
\hline & Association of G3BP2a with messenger ribonucleoprotein complexes & Rat & Angenstein et al., 2002 \\
\hline & Association of G3BP1 with stress granules & Mammalian cell lines & Tourriere et al., 2003 \\
\hline \multirow[t]{5}{*}{ Signal Transduction } & Interaction with the RasGAP pathway & Mammalian cell lines and Drosophila & $\begin{array}{l}\text { Parker et al., 1996, Kennedy et al., } 2001 \\
\text { Pazman et al., } 2000\end{array}$ \\
\hline & G3BP1 and Ras signaling (review) & & Tocque et al., 1997 \\
\hline & G3BP2 participation with IkappaBalpha /NF-kappa B signaling & HeLa cells & Prigent et al., 2000 \\
\hline & HER2 signaling and G3BP1 & Human & Barnes et al., 2002 \\
\hline & Protein kinase $\mathrm{C} \beta 2$ transduction and G3BP2a & Rat & Angenstein et al., 2002 \\
\hline Structural & $\begin{array}{l}\text { RRM, SH3 domain-binding motifs, acid-rich and RGG motifs } \\
\text { NTF2 motif }\end{array}$ & $\begin{array}{l}\text { Mammals } \\
\text { Human, C. Elegans, A. Thaliana, S. } \\
\text { Cerevisiae, S. Pombe }\end{array}$ & $\begin{array}{l}\text { Kennedy et al., 1997, Parker et al., } 1996 \\
\text { Suyama et al., } 2000\end{array}$ \\
\hline \multirow[t]{3}{*}{$\begin{array}{l}\text { Cancer progression or } \\
\text { maintenance }\end{array}$} & Demonstrated over-expression of G3BP1 in cancer specimens & Human tissues and cell lines & $\begin{array}{l}\text { Barnes et al., 2002, } \\
\text { Guitard et al., } 2001\end{array}$ \\
\hline & $\begin{array}{l}\text { Demonstrated changed expression of G3BP1 in other metastatic lung } \\
\text { and prostate }\end{array}$ & $\begin{array}{l}\text { Mammals/cell lines derived from metastatic } \\
\text { cancers }\end{array}$ & Liu et al., 2001 \\
\hline & Demonstrated over-expression of G3BP1/2 in human breast cancer & Human tissues & French et al., 2002 \\
\hline \multirow{3}{*}{$\begin{array}{l}\text { Developmental } \\
\text { aspects }\end{array}$} & De-differentiation of epithelial cells & human retinal pigment epithelial cells & Kociok et al., 1999 \\
\hline & Role in ommatidial polarisation & Drosophila & Pazman et al., 2000 \\
\hline & Suggested G3BP1 may have a role in neuronal differentiation & P19 cells & Atlas et al., 2004 \\
\hline Neurobiology & Maintenance of neural cells by G3BP1 & Rat and P19 cells & Angenstein et al., 2002, Atlas et al., 2004 \\
\hline \multirow[t]{3}{*}{ Cell cycle } & Proliferation of epithelial cells was correlated to G3BP1 & human retinal pigment epithelial cells & Kociok et al., 1999 \\
\hline & G3BP1 was correlated to S phase entry & Human cancers and Human derived cells & Guitard et al., 2001 \\
\hline & G3BP1 expression was shown to peak during mitosis & HeLa cells & Whitfield et al., 2002 \\
\hline \multirow[t]{3}{*}{$\begin{array}{l}\text { Sub-cellular } \\
\text { localisation }\end{array}$} & $\begin{array}{l}\text { G3BP2a was shown to retain IkappaBalpha and IkappaBalpha /NF- } \\
\text { kappa B complexes in the cytoplasm }\end{array}$ & HeLa cells & Prigent et al., 2000 \\
\hline & $\begin{array}{l}\text { Suggested that G3BP1/2a/2b interact with Ran at the nuclear pore } \\
\text { (review) }\end{array}$ & Mammalian & Macara, 2001 \\
\hline & Nuclear localization of G3BP1 in stimulated cells & Human & Barnes et al., 2002 \\
\hline Tissue specific & $\begin{array}{l}\text { Cell cycle dependent movement of G3BP2 into the nucleus } \\
\text { Characterization of G3BP1/2 in mouse tissues }\end{array}$ & $\begin{array}{l}\text { NIH cells } \\
\text { Mouse }\end{array}$ & $\begin{array}{l}\text { French et al., } 2002 \\
\text { Kennedy et al., } 2001\end{array}$ \\
\hline expression & & & \\
\hline Protein stability & Modulation of USP10/ Ubp3 by G3BP1 & Human and S. Cerevisiae & $\begin{array}{l}\text { Soncini et al., 2001, } \\
\text { Cohen et al., 2003a, } \\
\text { Cohen et al., 2003b. }\end{array}$ \\
\hline Human disorders & $\begin{array}{l}\text { Reduced G3BP1 mRNA in Fragile } X \text { cells } \\
\text { myelodysplastic syndromes (5q- syndrome, G3BP1 on } 5 q \text { ) }\end{array}$ & $\begin{array}{l}\text { Human } \\
\text { Human }\end{array}$ & $\begin{array}{l}\text { Zhong et al., 1999*} \\
\text { Boultwood et al., 2002* }\end{array}$ \\
\hline
\end{tabular}

* indicates articles not reviewed in the current manuscript 
1996; see also Fig. 2A). Co-immunoprecipitation of RasGAP and G3BP occurred only in growing cells and depended on Ras activation (Gallouzi et al., 1998, Parker et al., 1996). The RasGAPG3BP1 complex detected in proliferating cells distributed to the membrane fraction after cell lysis, consistent with the interpretation that both proteins are recruited to activated Ras (Gallouzi et al., 1998). Gallouzi and colleagues reported that G3BP1 was heavily serine phosphorylated in quiescent cells, where three isoelectric variants caused by differential phosphorylation were detected. Only two of these persisted in proliferating cells (Gallouzi et al., 1998). The phospho-peptide missing in proliferating cells was found to correspond to G3BP1 phosphorylation at Ser149 (Gallouzi et al., 1998). The same G3BP phospho-peptide was absent in RasGAP ${ }^{-/-}$fibroblasts regardless of proliferation state, suggesting cell-cycle regulated phosphorylation of Ser149 is RasGAP dependent (Tourriere et al., 2001). Ser149 is a consensus Casein Kinase II phosphorylation site $^{2}$ and is conserved between G3BP1 and the G3BP2 isoforms. Phosphotryptic peptide mapping revealed no differences in phosphorylation sites between quiescent and dividing cells (Gallouzi et al., 1998). It would thus appear that G3BP1 is constitutively phosphorylated in resting cells and serum stimulation induces its dephosphorylation, perhaps via recruitment of a specific phosphatase or inhibition of a specific kinase. G3BP1 phosphorylation and Ras-GTPdependent RasGAP association are consistent with the possibility that G3BP1 functions in response to external signals. Nevertheless, G3BP1 was able to associate with a purified RasGAP SH3 domain regardless of its phosphorylation status, implying that G3BP phosphorylation affects downstream functions rather than RasGAP interaction (Gallouzi et al., 1998). G3BP1 phosphorylation on Ser149 affects a number of functions so far assigned to the protein, including RNase activity and stress granule recruitment/assembly (see below for detailed discussion) (Tourriere etal., 2003, Tourriere etal., 2001). Human G3BP2a has also been demonstrated to associate with RasGAP in in vitrobead-binding assays (Kennedy et al., 2001). If all family members do function as RasGAP effectors in vivo they may mediate similar, opposing or completely different effects. SH3 domains are relatively promiscuous and subtle differences in the domains and their ligands can alter binding specificity (Agrawal and Kishan, 2002). The contextual differences between the PxxP motifs in the G3BPs could therefore be functionally relevant.

\section{G3BP1 may be regulated through HER}

G3BP was further, albeit indirectly, implicated in Ras signalling when it was found that G3BP1 mRNA is induced downstream of Human Epidermal Growth Factor Receptors (HER) (Barnes et al., 2002). mRNA differential display experiments in the SKBR3 breast cancer cell line revealed that G3BP1 is transcriptionally induced by Heregulin (HRG), a combinatorial ligand for HER3/4, and this was subsequently confirmed at the protein level. MCF7 breast cancer cells over-expressing HER2 also up-regulated G3BP1 expression (Barnes etal., 2002). These results are in contrast to previous studies which showed no regulation of G3BP1 in fibroblast cell lines stimulated by serum or Epidermal Growth Factor (Gallouzi et al., 1998, Parker et al., 1996). HRG stimulation of MCF7 cells also promoted G3BP1 phosphorylation, at an undetermined site and increased its association with RasGAP in distinct cytoplasmic granules (Barnes et al., 2002). HRG treatment also induced translocation of G3BP1 to the

Note 2: As predicted by Phosphobase, http://www.ebi.ac.uk nucleus in a small portion of cells. G3BP1 specifically co-localised with histone deacetylase $\mathrm{H} 3$, a marker for active transcription sites (Fig. 2G). The authors of this study concluded that G3BP1 was functioning as a Ras effector in this system. Their results, however, contradict the observations made in fibroblastic cells, where G3BP1 phosphorylation has been found to decrease in response to extracellular signals. Moreover, G3BP1 dephosphorylation was important for G3BP1 function in other systems (Gallouzi et al., 1998, Tourriere et al., 2003, Tourriere et al., 2001). The putative G3BP1 phosphorylation site was not identified in the Barnes et al. study, so these results may yet be reconcilable. Alternatively, there may be differences between G3BP1 functions in fibroblasts and breast cancer cells.

\section{G3BPs and cell cycle regulation}

Several studies implicate G3BP1 in cell cycle regulation. G3BP1 over-expression in fibroblasts was found to increase S-phase entry and this was dependent on an intact RNA-binding domain (Guitard et al., 2001). In addition, G3BP1 is up-regulated in proliferating Retinal Pigment Epithelial (RPE) cells, which are characteristic of proliferative vitreoretinopathy (PVR) (Kociok etal., 1999). A comprehensive microarray analysis of cell cycle regulated genes in HeLa cells identified periodic expression of G3BP1 throughout the cell cycle and classified it as a cell-cycle regulated transcript (Whitfield et al., 2002). In that study G3BP1 expression was found to peak in mitosis, along with clusters of genes known to function in actin cytoskeleton remodelling and the cell adhesion changes necessary for mitosis, in Ras signal transduction and in chromosome metabolism (Whitfield et al., 2002). Finally, G3BP1 may bind and regulate $c$ myc mRNA (see below). c-Myc is an important transcription factor predominantly involved in the regulation of cell cycle progression (Dang, 1999, Dang et al., 1999).

\section{G3BPs and Ras signalling in Drosophila}

Genetic studies have also implicated the Drosophila homologue of G3BP in Ras signal transduction. Drosophila encodes only one G3BP: Rasputin (Rin). Rin shares $40 \%$ identity and $60 \%$ similarity with mouse G3BP1 and 2 a over its entire length. ClustalW alignment of Rin with G3BP1 or G3BP2a generates almost identical similarity scores, indicating that Rin is not 'more similar' to G3BP1 or G3BP2. $\mathrm{Rin}^{-/-}$flies are viable and display defects in photoreceptor recruitment and ommatidial polarity in the eye (Pazman etal., 2000) (see below). The Drosophilaeye is widely used as a system for dissecting the Ras signalling pathway, since Ras is required for the differentiation of all photoreceptors (Simon et al., 1991). Rin genetically interacted with the Ras pathway with data indicating a role at the level of Ras or above and independent of Raf (Pazman etal., 2000). The phenotype of $\mathrm{Rin}^{-1-}$ flies was similar to RhoA mutants. This and other observations suggested a role for Rin as a link between Ras and Rho signalling, possibly in regulation and maintenance of the actin cytoskeleton (Pazman et al., 2000). Although Ras and Raf are required for cell proliferation in Drosophila, $\mathrm{Rin}^{-/-}$tissue proliferates (Pazman et al., 2000), so a role for Drosophila G3BP in Ras control of proliferation pathways is not supported.

\section{G3BPs and invertebrate development}

Cellular differentiation, development and polarisation of the ommatidial units in the Drosophilaeye imaginal disc has been well documented and used to dissect not only the Ras signalling 


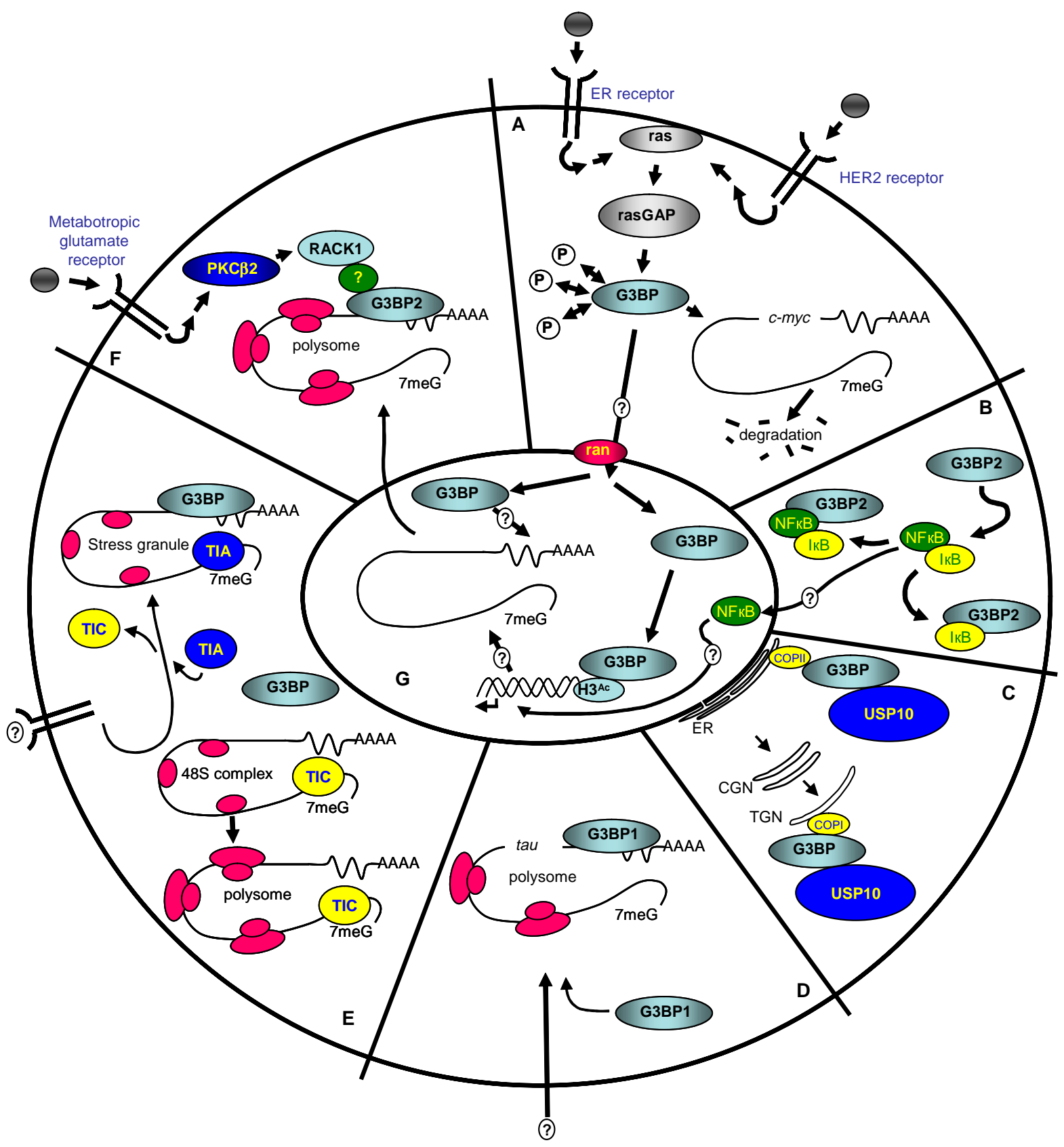

Fig. 2. Schematic representation of the putative biological activities of G3BPs in cells. The diagram encapsulates the various cytoplasmic activities that have been reported in the literature including: (A) rasGAP signalling, represented downstream of the ER and/or HER receptor and speculatively transducing to c-myc degradation; (B) involvement in NFKB and I $\kappa B$ nucleo-cytoplasmic equilibrium; (C) interactions with ubiquitin-specific proteases (USP) which may participate in retrograde and ante-retrograde protein secretion pathways. Various roles associated with RNA metabolism are also proposed, such as (D) which shows an interaction of G3BP1 with tau MRNA; (E) participation in stress granule formation and (F) a role, downstream of the metabotropic glutamate receptor, in polysome activity. (G) shows a possible role for G3BPs in the nucleus interacting with acetylated Histone 3 and speculatively with mRNA to facilitate export. G3BP is depicted as entering the nucleus via a ran-mediated pathway. Abbreviations: PKCB2 protein kinase C $\beta 2$; TIC, translation initiation complex; TIA, T-Cell internal antigen; ER, Endoplasmic reticulum; CGN, cis-Golgi network; TGN, trans-Golgi network.

pathway but the pathways that lead to polarisation which are conserved to a large degree in vertebrates (Strutt, 2003). In general, there are two distinguishable processes required for ommatidial polarisation. Both appear to occur concurrently during the movement of the morphological furrow across the imaginal disc that initiates differentiation of the eye and antenna. The first distinct event in ommatidial formation is the differentiation of the $R 8$ receptor followed by the differentiation of the R3/R4 photoreceptor pair, with R3 differentiation conferring chirality to the ommatidial unit (Fig. 3). The second event is the $90^{\circ}$ rotation of the 


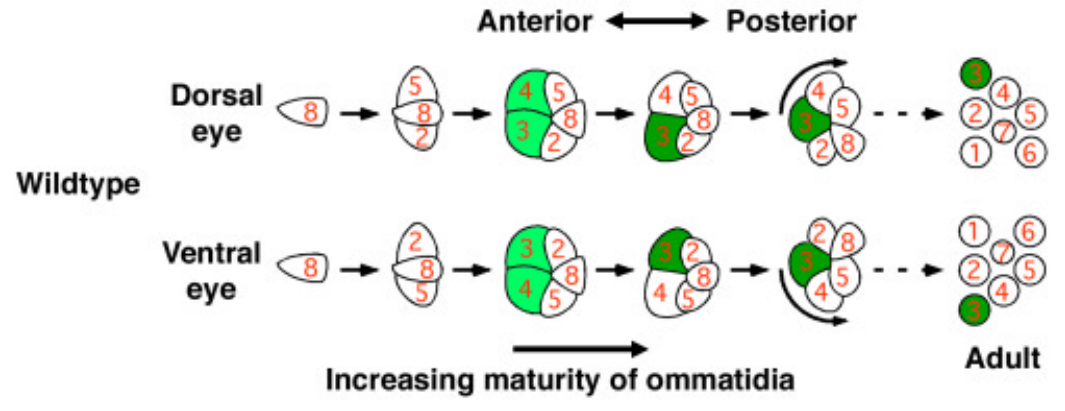

Fig. 3. Schematic representation showing planar polarisation of the ommatidia within the Drosophila eye. Initially cells are recruited to the cluster followed by polarisation of $R 8$ and subsequent specification of $R 3$ within the R3/R4 cell pair to determine chirality. Finally the ommatidia rotate so that the $R 3$ cell is orientated away from the equator of the imaginal disc. Reprinted with permission from Strutt, (2003). fates (Marlow etal., 2002). There appear to be three G3BP homologues $(\alpha, \beta$ and $\gamma)$ in zebrafish. We knocked down G3BP $\beta$ with specific morpholinos and observed a phenotype that suggests defects in $\mathrm{CE}$ and/or cell tracking with no apparent changes in cell fate $(\mathrm{N}=73$ with 60 abnormal phenotypes, control injections $=65$ with 0 abnormal phenotypes, Fig. 5). Full characterization of this data will be published elsewhere.

Another aspect of polarisation is that asymmetric distribution of the core proteins, involved in downstream pathways, is common within cells (Strutt, 2003) and this was also shown for Rin (Pazman et al., 2000). It is interesting to note that we also observed an asymmetric distribution of G3BP1 cells when studying its expression in human breast cancer sections (French et al., 2002, see also Fig. 6).

The classification of G3BP1 as a cell-cycleunit in such a manner that the R3 moves away from the equator of the disc (Fig. 3). Pazman and colleagues (Pazman et al., 2000) studied the effects of Rin mutants on ommatidial polarisation in Drosophila (Fig. 4 A-C) and concluded that changes in development were most similar to those of RhoA and other polarity genes such as frizzled $(f z)$ and dishevelled (dsh). Additional experiments using constructs driven by the sevenless promoter lead them to conclude that the Rin mutation genetically interacts with RhoA and proposed a signal transduction pathway that incorporated these findings (Fig. 4D).

\section{G3BPs and vertebrate development}

Recently evidence has emerged that several downstream effectors of planar polarisation in Drosophila are involved in Convergence and Extension (CE) in vertebrates and that the processes are broadly similar (reviewed in Strutt, 2003). Of particular interest is the study of Rho kinase 2 (Rok2) which suggests that Rok2 acts downstream of non-canonical Wnt11 to mediate mediolateral cell elongation which is in turn required for dorsal cell movement along straight paths without altering cell regulated transcript peaking in mitosis discussed above would also seem to support a role for G3BP in morphological remodelling and adhesion (Whitfield et al., 2002). It is also noteworthy in this regard that the $\mathrm{SH} 3$ domain of RasGAP has been linked to signalling Rho-mediated cytoskeletal re-organisation in mouse fibroblasts, although this activity was not dependent on Ras (Leblanc et al., 1998).

\section{G3BP2 and NFKB signalling}

The Nuclear Factor- $\mathrm{kB}(\mathrm{NF} \kappa \mathrm{B})$ family of transcription factors comprises five related proteins that act as homo- and heterodimers to regulate gene transcription. They are ubiquitous transcription factors and their capacity for potent and rapid induction of gene expression is extensively exploited (May and Ghosh, 1998). NFKB dimers are sequestered in the cytoplasm of unstimulated cells bound to inhibitory proteins called IKBs (Inhibitors of NFKB) (Whiteside and Israel, 1997). IKB $\alpha$ functions in the nucleus to dissociate NFkBs from DNA and re-export them. Its nuclear functions are primarily controlled by its ability to translocate to this compartment (Huang et al., 2000).
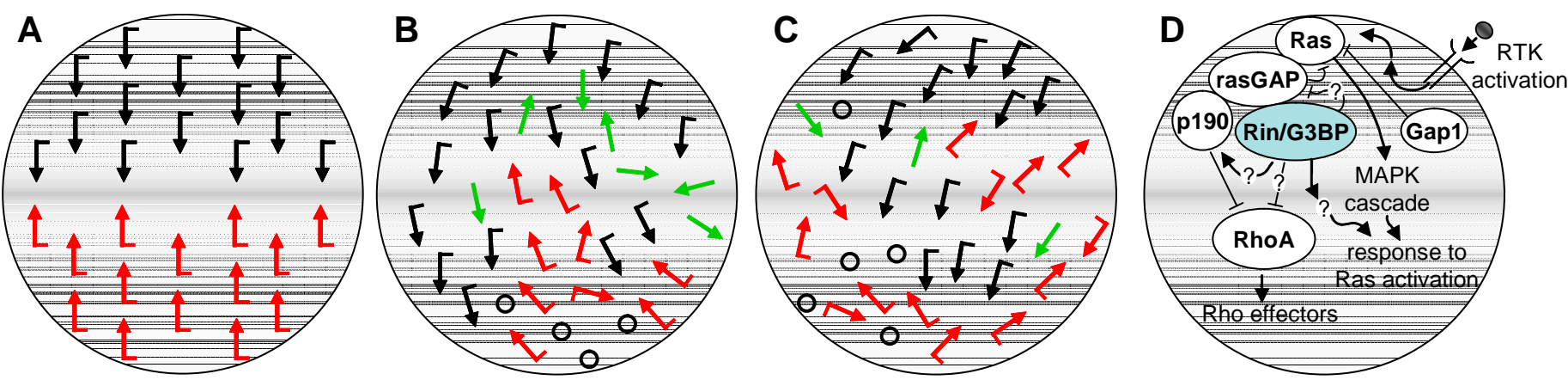

Fig. 4. Schematic representation of the adult eye phenotypes described in rin mutations by Pazman et al., (2000). Panels show tangential schematics of ommatidial polarity. Wild type (A) ommatidia are correctly polarised with respect to the equator, ventral ommatidia (red arrows) are mirror images of dorsal clusters (black). In $\mathrm{rin}^{1} / \mathrm{rin}^{1}$ and $\mathrm{rin}^{2} / \mathrm{rin}^{2}$ mutants (B,C) respectively, the ommatidial are not orientated correctly and display defects in the degree and direction of rotation, as well as chirality. Black arrows, dorsal chirality; red arrows, ventral identity; green arrows, non-chiral symmetrical ommatidia; black circles, ommatidia with recruitment defects (in these, polarity was not scored). (D) Schematic representation of the potential signal transduction pathways of Rin in Ras and Rho signaling. All figures adapted from Pazman et al., (2000). 


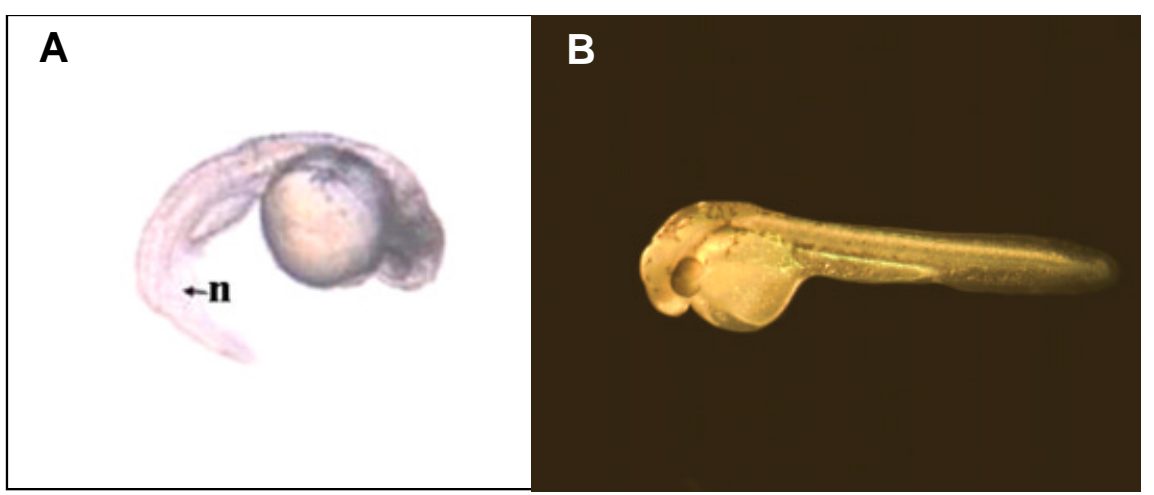

Fig. 5. Knockdown of zebrafish G3BP及 by specific morpholinos. (A) The phenotype shows a distinctive "kink" in the tail which has changed direction turning approximately $90^{\circ}$ ventrally. There is no apparent gross change in cell fate posterior to the kink. " $N$ " indicates the notochord and the position at which the tail is kinked. (B) A control zebrafish with no tail defects.

consequence of the interaction between $\mathrm{I} \kappa \mathrm{B} \alpha$ and G3BP2a could well depend on cellular activation status or the type of activating signal and post-translation modifications particular to these contexts.

\section{G3BPs and ubiquitin-mediated activity}

Ubiquitin-mediated protein degradation is a fundamental strategy used by cells to regulate protein turnover. In addition to the ubiquitinconjugating enzymes that attach poly-ubiquitin chains to lysine residues on substrates targeted for destruction, a number of de-ubiquitinating enzymes can catalyse the removal of ubiquitin, thereby providing another level of regulation. Ubiquitin-specific proteases (USPs) form a large family of proteases (there are more than 60

Each of the five NFKB family members possesses a conserved $\mathrm{N}$-terminal region, known as the Rel Homology Domain (RHD). This domain is responsible for $\mathrm{NF} \kappa \mathrm{B}$ dimerisation and DNA binding; as well as for $N F \kappa B$ interaction with I $\kappa B$ family members. Co-crystallisation studies on the $\mathrm{p} 50 / \mathrm{p} 65 \mathrm{NF} \kappa \mathrm{B}$ dimer in complex with I $\mathrm{KB} \alpha$ confirmed that the NLS of p50 remains exposed in this complex (Huxford et al., 1998). This suggested the presence of additional mechanisms accounting for the cytoplasmic localisation of NFkBs. Three such mechanisms have been proposed, one of which involves G3BP2 (Johnson et al., 1999; Huang et al., 2000). It has been suggested that I $\mathrm{kB} \alpha$ contains an $\mathrm{N}$-terminal cytoplasmic retention sequence, to which G3BP2 binds and promotes cytoplasmic retention (Prigent et al., 2000).

Prigent and colleagues identified a short cytoplasmic retention sequence (CRS) in I $\mathrm{kB} \alpha$ that was sufficient to promote retention of the protein in the cytoplasm of HeLa cells (Prigent et al., 2000). G3BP2a was identified as a CRS-binding protein, when the sequence was used as bait to discover interacting proteins. Coimmunoprecipitation experiments suggested that G3BP2a interacted with both $I \kappa B \alpha$ and $I \kappa B \alpha / N F \kappa B$ complexes and the ability of overexpressed G3BP2a to retain $1 \kappa B \alpha$ in the cytoplasm was demonstrated (Prigent etal., 2000) (Fig. 2B). The IkB $\alpha$ interacting domain on G3BP2a was mapped and the central acid-rich domain of G3BP2a (aa 117-223) was sufficient to promote cytoplasmic retention (Prigent et al., 2000). This interaction provides an intriguing link between G3BP2a and NFKB signalling, since it implies that G3BP2a may play a role in regulating the nucleocytoplasmic $\mathrm{NF} \kappa \mathrm{B} / \mathrm{l} \kappa \mathrm{B} \alpha$ equilibrium and therefore $\mathrm{NF} \kappa \mathrm{B}$ activity. The suggestion that G3BP2a interacts with both $1 \kappa B \alpha$ and $I \kappa B \alpha /$ $\mathrm{NF} \kappa \mathrm{B}$ complexes is somewhat paradoxical, however. G3BP2a retention of $I \kappa B \alpha$ alone in the cytoplasm implies a positive influence on NFKB activation, since IKB $\alpha$ would then be prevented from inhibiting NFKB in the nucleus. Conversely, G3BP2a-mediated cytoplasmic retention of $I \kappa B \alpha$ in complex with $\mathrm{NF} \kappa \mathrm{B}$ would imply a negative role in NFKB activation. Interestingly, I $\mathrm{KB} \alpha \mathrm{Tyr} 42$ phosphorylation conferred dramatically decreased CRS function (Prigent et al., 2000). These observations imply that signalinduced modification of the IKB $\alpha$ CRS could affect the affinity of CRS-binding partners, such as G3BP2a and therefore cytoplasmic retention of $I \kappa B \alpha$. The biological relevance of these interactions in an inducible system is yet to be determined. The real mammalian members) and are thought to be extremely substratespecific regulators of a variety of biological processes (Ciechanover et al., 2000, Hicke, 2001). The canonical proteasome-dependent function of ubiquitination involves poly-ubiquitination where ubiquitin subunits are linked through their Lys 48 residues. Ubiquitin is, however, also widely used as a reversible protein modification with diverse biological effects. Polyubiquitination through Lys63 residues on each ubiquitin subunit, for example, serves as a signal for the target to assemble with other proteins (Wilkinson, 2003). Mono-ubiquitination, in conjunction with ubiquitin-binding proteins, can signal changes in protein location, activity and interactions with binding partners (Schnell and Hicke, 2003).

Three publications indicate an interaction between G3BP1 and a de-ubiquitinating enzyme. In the first case an interaction between G3BP1 and Ubiquitin Specific Protease-10 (USP10) was discovered using a yeast-two-hybrid system and confirmed in human cells. G3BP1 did not appear to be a substrate of USP-10, rather, it inhibited USP-10 de-ubiquitinating activity on a linear ubiquitin construct in vitro (Soncini et al., 2001). Subsequently,

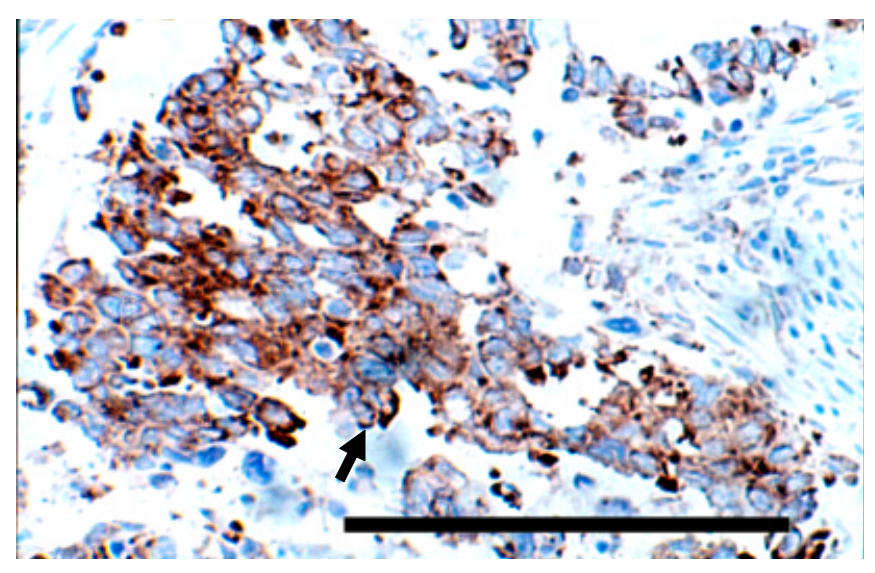

Fig. 6. Immunohistochemical staining of a human breast cancer (invasive ductal carcinoma) section stained with G3BP1-specific antibodies. The section shows extensive expression of G3BP1 in cancerous cells. The arrow indicates asymmetrical staining of G3BP1 in a cancerous cell. Reprinted with permission from French et al., (2002). 
the Saccromyces cerevisiaeG3BP1 homologue, Bre5, was found to be a necessary part of a Ubp3 (the yeast homologue of USP. 10) de-ubiquitinating complex. In contrast to the previous report, USP-10 required G3BP1 to form a de-ubiquinating complex in vivo (Cohen et al., 2003a, Cohen et al., 2003b) ${ }^{3}$. Two substrates for the USP10/G3BP1 de-ubiquitinating complex have been discovered so far: Sec23, a component of the COPII complex involved in anterograde protein export from the endoplasmic reticulum (ER) to the Golgi network (see Fig. $2 \mathrm{C}$ ) and $\beta$-COP, a component of the COPI complex required for retrograde protein transport from the Golgi to the ER. De-ubiquitination of Sec23 and $\beta$-COP rescues them from degradation by the proteasome, thereby maintaining the activity of the retrograde and anterograde protein secretion pathways (Cohen et al., 2003a, Cohen et al., 2003b) (Fig. 2C). The apparent contradiction between the two influences on the USP-10 de-ubiquitination complex reported, one activating and one inhibitory, has not been resolved. Since one of these studies employed a non-physiological ubiquitin substrate (Soncini et al., 2001) it has been suggested that G3BP1 might function in vivo to restrict de-ubiquitinating activity to appropriate substrates (Cohen etal., 2003a). USP-10 appears to be a mono-ubiquitin deubiquitinating enzyme, cleaving single ubiquitin molecules from protein substrates rather than poly-ubiquitin chains (Cohen et al., 2003a). Whilst the de-ubiquitination activity with which it has been associated so far involves rescuing substrates from proteasomal degradation, it appears to have multiple substrates (Cohen et al., 2003a) and could thus be involved in non-canonical forms of ubiquitin-mediated regulation.

With regard to the potential function/s of G3BP1 in ubiquitin metabolism, it is noteworthy that several studies have demonstrated roles for ubiquitination and the proteasome in mRNA degradation. G3BP1 has been ascribed an endoribonuclease function and thus been implicated in mRNA degradation (see below "G3BPs and RNA Metabolism" for detailed discussion) (Gallouzi etal., 1998, Tourriere etal., 2001). Isolated proteasomes have also been demonstrated to harbour a substrate specific endoribonuclease activity (Gautier-Bert et al., 2003). In one study, degradation of an ARE-containing reporter mRNA was regulated by the level of ubiquitin-conjugating activity in the cell and inhibition of a cytokine-inducible de-ubiquitinating enzyme enhanced mRNA decay (Laroia et al., 2002). The mRNA destabilising protein AUF1 is regulated by ubiquitination and inhibition of the proteasome or the ubiquitin-conjugating enzyme that acts on AUF1 completely blocks mRNA decay (Laroia et al., 1999, Laroia and Schneider, 2002). A large number of deubiquitinating enzymes exist, presumably to provide substrate specificity. Whether G3BP1's involvement in ubiquitin metabolism is linked to its involvement in mRNA metabolism, or other ubiquitin-mediated activities such as signal transduction, is unknown. G3BP1 may interact with de-ubiquitinating enzymes other than USP-10, USP-10 may have substrates other than the vesicle transport proteins mentioned above, or protein transport and mRNA metabolism could be mechanistically linked in a way that is not yet appreciated.

Note 3: Bre5 and Ubp3 will be referred to here by the names of their mammalian counterparts, G3BP1 and USP10, for the sake of clarity.

\section{G3BPs and RNA metabolism}

One of the most interesting features of the G3BPs is their propensity to bind RNA. Diverse mRNA-binding proteins compose transcript-specific RNA-protein complexes, termed messenger ribonucleoprotein particles (mRNPs). The mRNP is a dynamic ultrastructure that regulates all aspects of the life of an mRNA including nuclear processing, transport, translation and decay. Far from simply chaperoning mRNAs, it has significant effects on the coordination of gene expression (Keene, 2001, Keene and Tenenbaum, 2002). Post-transcriptional regulation always intersects with questions of whether, when and sometimes where messages are translated and it is the mRNP ensemble, rather than the mRNA itself that determines temporal and spatial regulation of mRNA translation. Alterations to mRNP composition or modifications of $\mathrm{mRNP}$ components are responsible for $\mathrm{mRNA}$ receptivity to extracellular signals. RBPs may have affinity for one, several or, in the case of general factors such as poly $(A)$-binding protein, almost all mRNAs. The function of G3BPs in the mRNP environment is not clear, nor have their in vivoligands been characterised. The studies that have examined the intersection of G3BPs with RNA metabolism, outlined in this section, suggest that G3BPs may regulate mRNA translation or decay, or both.

\section{G3BPs and mRNA stability}

The likely RNA-binding and protein interaction domains of the G3BPs have prompted speculation that they are involved in signal-regulated mRNA metabolism. Although all G3BPs bind homopolymeric ribonucleic acid sequences in vitro (Tourriere et al., 2001) and G3BP1 has been immunoprecipitated with a heterogenous pool of polyA mRNAs (Tourriere et al., 2001), c$m y c$ is the only specific mRNA to which they have been shown to bind, also in vitro (Gallouzi et al., 1998, Tourriere et al., 2001).

Intriguingly, G3BP1 demonstrated a phosphorylation-dependent endoribonuclease activity on the c-myc 3'UTR in vitro, despite the absence of any identifiable ribonuclease domain (Gallouzi et al., 1998, Tourriere et al., 2001). G3BP2a and G3BP2b also mediate $c$-myc cleavage in vitro, although with much lower efficiency than G3BP1 (unpublished data). As mentioned earlier, G3BP1 is heavily serine phosphorylated in quiescent cells and dephosphorylated in growth-factor stimulated cells (Gallouzi et al., 1998). This cell-cycle-dependent regulation of G3BP1 RNase activity suggested to the authors that G3BP1 might function as a growth factor sensor, allowing accumulation of transcripts involved in cell cycle regulation, such as $c-m y c$, in stimulated cells; but facilitating their degradation in resting cells. This is a plausible hypothesis, since mitogen-activated pathways are well known to exert post-transcriptional effects on mRNA stability and translation (Ross, 1995). Further support for this hypothesis lies in the fact that $c$-myc mRNA decay is delayed in

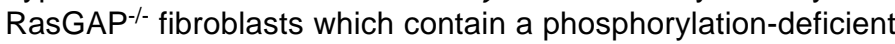
form of G3BP1 (Tourriere et al., 2001). Subsequent to the initial identification of G3BP1 as an RNase, Tourriere and co-authors showed specific cleavage of $c$-myc between CA di-nucleotides, which was dependent on RNA-binding; and determined an affinity binding sequence for G3BP1 using the SELEX technique (Tourriere et al., 2001). A range of mRNAs showed exact matches to the experimentally-determined G3BP1 consensus binding sequence, including other cell-cycle regulators (Tourriere et al., 2001). 
The mechanisms and regulation of MRNA degradation have been intensively studied over recent years. A small number of general pathways appear to be responsible for degrading most mRNAs, so regulatory mechanisms are thought to be targeted to the initial events that direct the mRNA into one of these pathways, maintain it in a translationally inactive state, or permit its translation (Jacobson and Peltz, 1996). The circular structure of the mRNP is paramount since it is thought to be this structure that is translationally competent, in the absence of other factors preventing translation initiation. Disruption of this structure frequently signals the initiation of mRNA decay and promotes the recruitment of a large macromolecular machine (the exosome) that subsequently degrades the body of the mRNA (Chen etal., 2001). The three major decay initiating events are deadenylation, endonucleolytic cleavage and decapping. Deadenylation-dependent mechanisms, such as decay directed by the prototypical AU-Rich element (ARE), have been the most widely studied and may be the predominant initiating event (Decker and Parker, 2002).

$C$-myc transcript levels, which G3BP1 has been proposed to regulate, are subject to post-transcriptional regulation by both deadenylation-dependent and-independent mechanisms (Brewer, 2000), including by endonucleolytic cleavage. It is well known that $c$-myc expression is instrumental in maintaining equilibrium between cell proliferation and differentiation (Dang, 1999, Dang et al., 1999), but the particular biological significance of G3BP1mediated $c$-myc decay is not clear. In addition to the G3BP1 binding site in the $c$-myc 3 ' UTR a 249 nt coding region instability determinant (CRD) sequence has been well studied. The endoribonuclease that cleaves $c$-myc in this region has been identified (Lee et al., 1998), as has a protein that protects $c$-myc from degradation by binding in the same region (Lemm and Ross, 2002). C-myc also harbours an ARE and has been shown to be degraded by a deadenylation-dependent mechanism (Brewer and Ross, 1988) and activation of the deadenylation-dependent or -independent pathway appears to be stimulus and cell type specific (Brewer, 2000).

Although numerous endonucleolytic cleavage events have been detected, very few of the enzymes that actually cleave target mRNAs have been cloned. Whether G3BP1 (and possibly G3BP2) endonuclease activity is relevant in vivo and whether it is limited to $c$-myc, remains to be resolved. The co-immunoprecipitation of G3BP1 with a pool of mRNAs (Tourriere et al., 2001) could suggest the existence of multiple G3BP1 ligands; however, it could also reflect heterogeneous mRNP membership independent of direct RNA binding.

\section{G3BPs are components of various mRNPs}

The main evidence that G3BP2 isoforms bind RNA in vivocomes from the isolation of G3BP2a in an mRNP complex associated with actively translated mRNAs in neuronal synapses (Angenstein et al., 2002). This complex also contained activated Protein Kinase C- $\beta 2$ (PKC- 32 ) and its receptor RACK-1 (Fig. 2F) (Receptor for Activated C Kinase-1). PKC activation induced PKC- $\beta 2 /$ RACK-1 association with actively translating polysomes, implying that protein synthesis of certain mRNAs in these neuronal synapses is signal regulated. RACK-1 and related proteins mediate the rapid subcellular distribution of PKC isoforms from the cytosol to other cellular compartments, including the cytoskeleton, Golgi complex and now mRNPs. This serves as a mechanism for PKC access to its substrates. PKC is activated in a range of cell responses depending on cell type and specific isoform activation, can influence cell proliferation, differentiation and cytoskeletal rearrangement (Ciesielski-Treska et al., 1991). In the experiment described above, PKC phosphorylated a subset of mRNA-associated proteins (Angenstein etal., 2002). G3BP2 isoforms contain a number of PKC consensus phosphorylation motifs ${ }^{2}$, but G3BP2a phosphorylation was not investigated in this study. Since RACK-1 does not have an RNA-binding domain, Angenstein and colleagues sought to identify the mRNP proteins that mediated mRNA binding. G3BP2a was a candidate for this activity, since its association with the mRNP was sensitive to RNase treatment (Angenstein et al., 2002). The role G3BP2a plays in this mRNP complex was not defined, nor is it known whether G3BP2a is a substrate for PKC, or whether such a complex is formed in cells other than neurons. However, this is the first evidence that G3BP2a plays a role in vivo in mRNA metabolism, as a member of a polysomeassociated mRNP.

G3BP1 was recently isolated as a member of an mRNP complex containing taumRNA (Fig. 2D), the mammalian ELAV protein HuD and insulin-like growth factor mRNA-binding protein IMP-1, which was formed during retinoic acid induced differentiation of $\mathrm{P} 19$ neuronal cells (Atlas et al., 2004). The interactions within the mRNP were RNA-dependent and the complexes co-sedimented with polysomal proteins, suggesting a role for this complex in tau translation. TaumRNP composition changed as P19 differentiation progressed. G3BP1 precipitated tau mRNA early in the differentiation process, thereby implicating the protein in the initial phases of mRNP assembly and regulation of expression (Atlas et al., 2004). Tau is a microtubule-associated protein important for maintaining microtubule stability and which is highly regulated during neuronal cell differentiation in order to allow for outgrowth and stability of neurites (Avila et al., 2004). This data not only shows G3BP1 is a polysomeassociated protein with a role to play in mRNA metabolism, but its presence in an mRNP containing taumRNA raises the possibility that it is through association with specific mRNAs that G3BP may participate in cytoskeletal re-modelling, as previously discussed.

Our own data studying the expression of G3BPs during mouse brain development (Fig. 7) suggests that all G3BPs are expressed during development with G3BP1 and G3BP2b rapidly down regulated at birth. A more detailed study is required to determine if the observed down regulation is true for all neural tissues. However, our histochemical data would suggest that G3BP1 is not expressed in mature neurons (Kennedy et al., 2001). This raises the question of whether G3BP1 is required for the maturation of neurons, especially

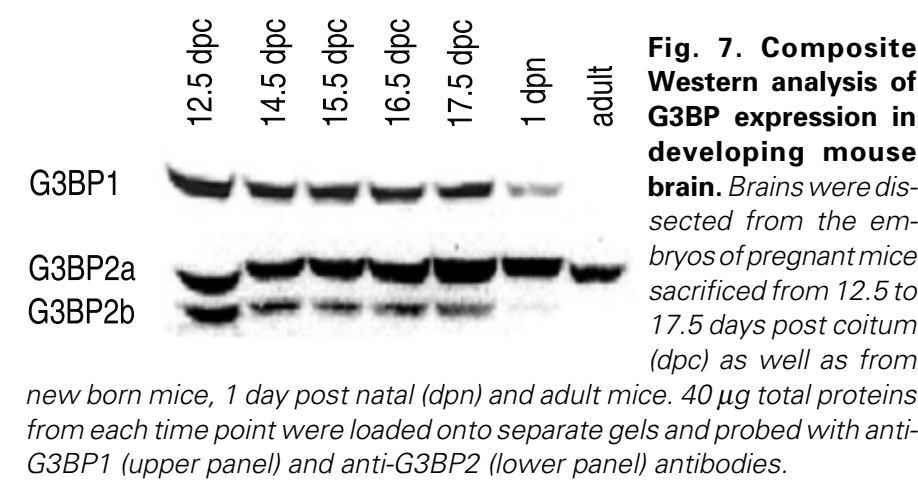


in light of the findings by Atlas and co-workers (Atlas et al., 2004).

A particularly interesting report implicated G3BP1 in stress granule assembly (Tourriere et al., 2003). Stress granules are large mRNA-containing complexes formed in the cytoplasm in response to a variety of cellular stresses (Fig. 2E). They are storage sites for abortive translation initiation complexes that can be further directed to translation initiation or decay pathways (Kedersha and Anderson, 2002). G3BP1 was recruited to stress granules after arsenite treatment of Cos or HeLa cells and G3BP1 over-expression was sufficient to induce the formation of stress granules in these cells (Tourriere et al., 2003). G3BP1 is dephosphorylated as a result of arsenite stimulation and this modification is essential for stress granule recruitment and assembly (Tourriere etal., 2003). The relevant phosphorylation site, Ser149, is the same site that is de-phosphorylated downstream of RasGAP (Gallouzi et al., 1998), thus implicating Ras activation in stress granule formation for the first time. Apart from translation initiation factors and small ribosomal subunits, other proteins that have been shown to function in mRNA metabolism are found in stress granules. These include T-cell internal antigen-1 (TIA-1) and T-cell internal antigen-1 related protein (TIAR), polyA binding protein (PABP), HuR and Tristetraprolin (TTP) (Fig. 2E) (Kedersha and Anderson, 2002). Stress granules have been postulated as sites of mRNA sorting at which the structure and composition of individual mRNPs determine whether messages are repacked and made translationally competent or degraded. It was thus suggested that G3BP1 might function to determine the fate of mRNAs during cellular stress. Preliminary data suggest that G3BP2 isoforms also have a role to play in stress granule formation since overexpressed G3BP1, 2a and 2b each accumulate in distinct cytoplasmic granules in a small percentage of transfected HEK293T cells (unpublished data). Conditions of stress have been reported to stabilise ARE mRNAs (reviewed in Gallouzi et al., 2000) and the evidence so far suggests this may be mediated through changes in ubiquitination state, localisation and interactions between a variety of RBPs.

The organization of mRNAs into mRNPs is raised to a new level when it is considered that mRNPs themselves are not randomly distributed throughout the cytoplasm. Indeed the diffusion of most cellular proteins and macromolecules appears to be severely limited (Hudder et al., 2003). mRNPs and components of the translation apparatus in particular may be anchored in the cytoplasm via interactions with cytoskeletal networks (Jansen, 1999). For example in neuronal cells the interaction of ELAV proteins with the cytoskeleton is either vital for delivery of transcripts to the polysome or conversely, ribosome-mRNA complex formation depends upon cytoskeletal components (Antic and Keene, 1998). Regulated transport, translation and stability of mRNA transcripts may thus be mediated by "linker" RBPs, like the ELAV and G3BP families, which act through association with the cytoskeleton and the polysome. It is interesting to note in this regard that G3BP1 was associated with an active Colony-Stimulating Factor-1 (CSF-1) receptor complex in macrophages and this complex also contained cytoskeletal components (Yeung et al., 1998).

\section{G3BP expression in cancer}

Both G3BP1 and G3BP2 are dramatically overexpressed in human cancers, in particular breast cancers (Barnes et al., 2002,
French et al., 2002, Guitard et al., 2001). Guitard and co-authors demonstrated over-expression of G3BP1 in a range of human tumours, including breast, head and neck, colon and thyroid (Guitard et al., 2001). Barnes and co-authors, having demonstrated G3BP1 induction downstream of HER2, tested eight human breast cancers and found G3BP1 to be overexpressed in all of them as compared to normal breast tissue from the same patients (Barnes et al., 2002). HER2 is also frequently overexpressed in breast cancer and this is associated with poor prognosis and malignancy (Yarden, 2001). G3BP1 over-expression paralleled HER2 over-expression in all tumours tested in that study (Barnes et al., 2002). We have demonstrated over-expression of G3BP2 in $88 \%$ of 56 breast tumours, whilst G3BP2 expression was rarely detectable in surrounding normal tissue (French et al., 2002). None of the above studies found any correlation between G3BP over-expression and clinicopathological parameters assigned to tumours such as histological grade, invasiveness or hormone receptor status.

These observations further implicate G3BPs in pathways that control cell proliferation and survival, amongst others, since these pathways are often disturbed in tumour cells. It is not yet known whether G3BPs function in tumour progression and by what mechanism, or whether they are simply up-regulated as a consequence of cancer. However, we have observed G3BP2 overexpression in early in situductal carcinomas, suggesting that the high expression of G3BP2 occurs in parallel with tumour progression rather than as a consequence of cancer formation (French et al., 2002). It is also noteworthy that reduced G3BP1 mRNA was found in metastatic compared to non-metastatic cells derived from human giant cell carcinoma of lung (Liu et al., 2001). This would appear to be inconsistent with data which suggest G3BPs are upregulated during cancer progression, but is nevertheless interesting given the fact that G3BP has been implicated in various pathways involving cytoskeletal dynamics and it is well documented that tumour metastasis is mediated through cytoskeletal rearrangement (Hanahan and Weinberg, 2000, Schmitz et al., 2000).

Accumulating evidence suggests that deregulated RNA processing is often associated with cell proliferation and cancer (Sonenberg and Gingras, 1998, Sueoka et al., 1999). Pathological stabilisation of ARE mRNA is often seen in tumour cells and TTP, an RBP which acts to destabilise ARE mRNA, has been shown to be a potent tumour suppressor (Stoecklin et al., 2003). G3BPs participate in mRNA metabolism and cell-cycle regulation and have been implicated in several signalling pathways involved in cancer, including Ras signalling (Malumbres and Pellicer, 1998), NFkB signalling (Chen and Goeddel, 2002) and the ubiquitin proteasome system (Gray et al., 1995). The evidence suggesting that RNA processing plays a key role in cancer progression and the observation that G3BPs are specifically overexpressed in a range of cancers, makes them candidate targets for anti-cancer therapeutics.

\section{Summary}

The foregoing discussion highlights what G3BP's domain structure initially suggested; that G3BPs are "scaffolding" proteins linking signal transduction to RNA metabolism. Whilst it is most attractive to hypothesise about G3BP's role in signalling to 
mRNA metabolism, it is not known whether all G3BP functions impinge on their RNA-binding activities, so any theories are naturally subject to this qualification. It is hypothesised that, in coordination with an array of other proteins, G3BP, in a phosphorylation-dependent manner, is involved in the post-transcriptional regulation of a subset of mRNAs, at least some of which are in common with those regulated by Hu proteins. These transcripts, partially controlled at the post-transcriptional level by G3BPs, code for proteins important in transcription (e.g. c-Myc) and cytoskeletal arrangement (e.g. Tau), amongst other as yet undetermined pathways. The subtle differences between G3BP family members could dictate binding to a variety of signalling proteins, so each of the G3BPs may participate in different, though possibly related mRNPs, which are assembled in response to different stimuli. The combinatorial nature of the mRNP complex offers a powerful means of regulating gene expression, beyond that provided by a simple mRNA sequence. The ways in which mRNP flexibility and specificity may be harnessed to coordinate gene expression of functionally or structurally related mRNAs are not yet fully appreciated. Characterising mRNP composition and the function/s of mRNP components, such as the G3BPs, will aid in the understanding of how post-transcriptional mechanisms contribute to the global regulation of gene expression.

\section{Acknowledgements}

We would like to acknowledge the members of the laboratory, Belinda Hartmann, Matthew Walker-Brown and Kerry Inder, who do not appear in the authorship of this paper but indirectly contributed to this manuscript. We also acknowledge Dr. Juliet French and Prof. Brian Key for the use of the zebrafish morpholino knockdown figure. Renee Stirling is supported by a Queensland Cancer Fund scholarship.

Note added in proof: Since the submission of this review, two articles (Cande et al., 2004; Hua and Zhou, 2004) have been published implicating a role of G3BP in stress granules. These articles have not been reviewed here.

\section{References}

AGRAWAL, V. and KISHAN, K. (2002). Promiscuous binding nature of sh3 domains to their target proteins. Protein Pept Lett. 9: 185-93.

ANGENSTEIN, F., EVANS, A., SETTLAGE, R., MORAN, S., LING, S., KLINTSOVA, A., SHABANOWITZ, J., HUNT, D. and GREENOUGH, W. (2002). A receptor for activated $\mathrm{c}$ kinase is part of messenger ribonucleoprotein complexes associated with polya-mrnas in neurons. JNeuroscience 22: 8827-8837

ANTIC, D. and KEENE, J. (1998). Messenger ribonucleoprotein complexes containing human elav proteins: Interactions with cytoskeleton and translational apparatus. J. Cell Science 111: 183

ATLAS, R., BEHAR, L., ELLIOTT, E. and GINZBURG, I. (2004). The insulin-like growth factor mrna binding-protein imp-1 and the ras-regulatory protein g3bp associate with tau mrna and hud protein in differentiated p19 neuronal cells. JNeurochem89: 613-26.

AVILA, J., LUCAS, J., PEREZ, M. and HERNANDEZ, F. (2004). Role of tau protein in both physiological and pathological conditions. Physiol Rev84: 361-84

BARNES, C.J., LI, F., MANDAL, M., YANG, Z., SAHIN, A.A. and KUMAR, R. (2002). Heregulin induces expression, atpase activity and nuclear localization of $\mathrm{g} 3 \mathrm{bp}$, a ras signaling component, in human breast tumors. Cancer Res 62: 1251-5.

BIRNEY, E., KUMAR, S. and KRAINER, A.R. (1993). Analysis of the rna-recognition motif and rs and rgg domains: Conservation in metazoan pre-mrna splicing factors. Nucleic Acids Res 21: 5803-16.

BOOKER, G., GOUT, I., DOWNING, A., DRISCOLL, P., BOYD, J., WATERFIELD, M. and CAMPBELL, L. (1993). Solution structure and ligand-binding site of the sh3 domain of the p85-alpha subunit of phosphatidylinositol 3-kinase. Ce//73: 813-822.

BREWER, G. (2000). Regulation of c-myc mrna decay in vitro by a phorbol esterinducible, ribosome-associated component in differentiating megakaryoblasts. $J$ Biol Chem 275: 33336-33345.

BREWER, G. and ROSS, J. (1988). Poly(a) shortening and degradation of the 3' a+urich sequences of human c-myc in a cell free system. Mol Cell Bio/8: 1697-1708.

BURD, C. and DREYFUSS, G. (1994). Conserved structures and diversity of functions of rna-binding proteins. Science 265: 615

CHEN, C., GHERZI, R., ONG, S., CHAN, E., RAIJMAKERS, R., PRUIJN, G., STOECKLIN, G., MORONI, C., MANN, M. and KARIN, M. (2001). Au binding proteins recruit the exosome to degrade are-containing mrnas. Cel/107: 451-464.

CHEN, G. and GOEDDEL, D. (2002). Tnf-r1 signaling: A beautiful pathway. Science 296: 1634-35.

CIECHANOVER, A., ORIAN, A. and SCHWARZ, A. (2000). Ubiquitin-mediated proteolysis: Biological regulation via destruction. Bioessays 22: 442-451.

CIESIELSKI-TRESKA, J., ULRICH, G. and AUNIS, D. (1991). Protein kinase c-induced redistribution of the cytoskeleton and phosphorylation of vimentin in cultured brain macrophages. JNeurosci Res 29: 362-78.

COHEN, M., STUTZ, F., BELGAREH, N., HAGUENAUER-TSAPIS, R. and DARGEMONT, C. (2003a). Ubp3 requires a cofactor, bre5, to specifically deubiquitinate the copii protein, sec23. Nat Cell Bio/5: 661-667.

COHEN, M., STUTZ, F. and C., D. (2003b). Deubiquitination, a new player in Golgi to endoplasmic reticulum retrograde transport. J Biol Chem. 278: 51989-92.

COSTA, M., OCHEM, A., STAUB, A. and FALASCHI, A. (1999). Human DNA helicase viii: A DNA and rna helicase corresponding to the g3bp protein and element of the ras transduction pathway. Nucleic Acids Res. 27: 817-821.

DANG, C. (1999). C-myc target genes involved in cell growth, apoptosis and metabolism. Mol Cell Biol. 19: 1-11.

DANG, C., RESAR, L., EMISON, E., KIM, S., LI, Q., PRESCOTT, J., WONSEY, D. and ZELLER, K. (1999). Function of the c-myc oncogenic transcription factor. Exp Cell Res. 253: 63-77.

DECKER, C.J. and PARKER, R. (2002). Mrna decay enzymes: Decappers conserved between yeast and mammals. Proc Nat/ Acad Sci USA 99: 12512-4

DUCHESNE, M., SCHWEIGHOFFER, F., PARKER, F., CLERC, F., FROBERT, Y THANG, M. and TOCQUE, B. (1993). Identification of the sh3 domain of gap as an essential sequence for ras-gap-mediated signaling. Science 259: 525-528.

FRENCH, J., STIRLING, R., WALSH, M. and KENNEDY, D. (2002). The expression of ras-gtpase activating protein sh3 domain-binding proteins, g3bps, in human breast cancers. Histochem J. 34: 223-231.

GALLOUZI, I., PARKER, F., CHEBLI, K., MAURIER, F., LABOURIER, E., BARLAT, I. CAPONY, J., TOCQUE, B. and TAZI, J. (1998). A novel phosphorylation-dependent rnase activity of gap-sh3 binding protein: A potential link between signal transduction and rna stability. Mol. Cell Biol. 18: 3956-3965.

GALLOUZI, I.E., BRENNAN, C.M., STENBERG, M.G., SWANSON, M.S., EVERSOLE A., MAIZELS, N. and STEITZ, J.A. (2000). Hur binding to cytoplasmic mrna is perturbed by heat shock. Proc Natl Acad Sci USA 97: 3073-8.

GAUTIER-BERT, K., MUROL, B., JAROUSSE, A., BALLUT, L., BADAOUI, S., PETIT, F. and SCHMID, H. (2003). Substrate affinity and substrate specificity of proteasomes with rnase activity. Mol Biol Reports 30: 1-7.

GHISOLFI, L., JOSEPH, G., AMALRIC, F. and ERARD, M. (1992). The glycine-rich domain of nucleolin has an unusual supersecondary structure responsible for its rna-helix-destabilizing properties. J Biol Chem 267: 2955-59.

GIGOUX, V., L'HOSTE, S., RAYNAUD, F., CAMONIS, J. and GARBAY, C. (2002). Identification of aurora kinases as rasgap src homology 3 domain-binding proteins. $J$ Biol Chem. 277: 23742-6.

GRAY, D., INAZAWA, K., GUPTA, A., WONG, R., UEDA, R. and TAKAHASHI, T. (1995). Elevated expression of unph, a proto-oncogene at 3p21.3, in human lung tumors. Oncogene 10: 2179-2183.

GUITARD, E., PARKER, F., MILLON, R., ABECASSIS, J. and TOCQUE, B. (2001) G3bp is overexpressed in human tumours and promotes s- phase entry. Cancer Lett. 162: 213-221.

HANAHAN, D. and WEINBERG, R.A. (2000). The hallmarks of cancer. Cel/100: 57-70 HICKE, L. (2001). A new ticket for entry into budding vesicles - ubiquitin. Cel/106: 527530 
HUANG, T., KUDO, N., YOSHIDA, M. and MIYAMOTO, S. (2000). A nuclear export signal in the n-terminal regulatory domain of ikappabalpha controls cytoplasmic localization of inactive nf-kappab/ikappabalpha complexes. Proc Nat/ Acad SCI USA 97: 1014-1019.

HUDDER, A., NATHANSON, L. and DEUTSCHER, M. (2003). Organization of mammalian cytoplasm. Mol Cel/ Bio/23: 9318-9326.

HUXFORD, T., HUANG, D., MALEK, S. and GHOSH, G. (1998). The crystal structure of the ikappabalpha/nf-kappab complex reveals mechanisms of nf-kappab inactivation. Cel/95: 759-770.

JACOBSON, A. and PELTZ, S. (1996). Interrelationships of the pathways of mrna decay and translation in eukaryotic cells. Annu. Rev. Biochem. 65: 693-739.

JANSEN, R. (1999). Rna-cytoskeletal associations. FASEB J13: 455-466.

JOHNSON, C., VAN ANTWERP, D. and HOPE, T. (1999). An n-terminal nuclear export signal is required for the nucleocytoplasmic shuttling of ikappabalpha. EMBOJ.18: 6682-93.

KEDERSHA, N. and ANDERSON, P. (2002). Stress granules: Sites of mrna triage that regulate mrna stability and translatability. Biochem. Soc. Trans. 30: 963.

KEENE, J. (2001). Ribonucleoprotein infrastructure regulating the flow of genetic information between the genome and the proteome. Proc. Natl. Acad. Sci. USA98: 7018-24.

KEENE, J. and TENENBAUM, S. (2002). Eukaryotic mrnps may represent posttranscriptional operons. Mol Cell. 9: 1161-7.

KENNEDY, D., FRENCH, J., GUITARD, E., RU, K., TOCQUE, B. and MATTICK, J. (2001). Characterisation of g3bps: Tissue specific expression, chromosomal localisation and rasgap120 binding studies. J. Cell Biol. 84: 173-187.

KENNEDY, D., RAMSDALE, T., MATTICK, J. and LITTLE, M. (1996). An rna recognition motif in wilms' tumour protein (wt1) revealed by structural modelling. Nat Genet 12: 329-31.

KENNEDY, D., WOOD, S., RAMSDALE, T., TAM, P., STEINER, K. and MATTICK, J. (1997). Identification of a mouse homolog of the human ras-gap-sh3-domain binding protein and structural confirmation that these proteins contain an rna recognition motif. Biomed. Pept. Prot. \& Nuc. Acids 2-3: 93-99.

KOCIOK, N., ESSER, P., UNFRIED, K., PARKER, F., SCHRAERMEYER, U., GRISANTI, S., TOQUE, B. and HEIMANN, K. (1999). Upregulation of the ras-gtpase activating protein (gap)-binding protein (g3bp) in proliferating rpe cells. $J$ Cell Biochem 74: 194-201.

LAROIA, G., CUESTA, R., BREWER, G. and SCHNEIDER, R.J. (1999). Control of mrna decay by heat shock-ubiquitin-proteasome pathway. Science 284: 499-502.

LAROIA, G., SARKAR, B. and SCHNEIDER, R. (2002). Ubiquitin-dependent mechanism regulates rapid turnover of au-rich cytokine mrnas. Proc. Natl. Acad. Sci. USA 99: $1842-1846$

LAROIA, G. and SCHNEIDER, R. (2002). Alternate exon insertion controls selective ubiquitination and degradation of different auf1 protein isoforms. Nucleic Acids Res 30: 3052-8.

LEBLANC, V., DELUMEAU, I. and TOCQUE, B. (1999). Ras-gtpase activating protein inhibition specifically induces apoptosis of tumour cells. Oncogene 18: 4884-4889.

LEBLANC, V., TOCQUE, B. and DELUMEAU, I. (1998). Ras-gap controls rhomediated cytoskeletal reorganisation though its sh3 domain. Mol. Cell Biol.18: 5567-78.

LEE, C., LEEDS, P. and ROSS, J. (1998). Purification and characterisation of a polysome-associated endoribonuclease that degrades c-myc mrna in vitro. J Biol Chem 273: 25261-25271

LEMM, I. and ROSS, J. (2002). Regulation of c-myc mrna decay by translational pausing in a coding region instability determinant. Mol Cel/ Bio/22: 3959-3969.

LIU, Y., DETH, R. and DEVYS, D. (1997). Sh3 domain-dependent associaton of huntingtin with epidermal growth factor receptor signaling complexes. J. Biol. Chem. 272: 8121-24.

LIU, Y., ZHENG, J., FANG, W., YOU, J., WANG, J., CUI, X. and WU, B. (2001) Identification of metastasis associated gene g3bp by differential display in human cancer cell sublines with different metastatic potentials g3bp as highly expressed in non-metastatic. Chin Med J (Engl) 114: 35-8.

MACARA, I. (2001). Transport into and out of the nucleus. MicrobiolMol. Biol. Rev. 65: 570.

MALUMBRES, M. and PELLICER, A. (1998). Ras pathways to cell cycle control and cell transformation. Front. Biosci. 3: 887-912.
MARLOW, F., TOPCZEWSKI, J., SEPICH, D. and SOLNICA-KREZEL, L. (2002). Zebrafish rho kinase 2 acts downstream of wnt 11 to mediate cell polarity and effective convergence and extension movements. Curr Bio/12: 876-84.

MAY, M. and GHOSH, S. (1998). Signal transduction through nf-kb. Immunology Today 19: 80

MAYEDA, A., MUNROE, J., CACERES, J. and KRAINER, A.R. (1994). Function of conserved domains of hnrnp a1 and other hnrnp a/b proteins. EMBO J. 13 : 5483-5495.

MEDEMA, R., DE LAAT, W., MARTIN, G., MCCORMICK, F. and BOS, J. (1992) Gtpase-activating protein sh2-sh3 domains induce gene expression in a rasdependent fashion. Mol Cel/ Biol12: 3425-30.

NICHOLS, R., WANG, X., TANG, J., HAMILTON, J., HIGH, F., HERSCHMAN, H. and RIGBY, W. (2000). The rgg domain in hnrnpa2 affects subcellular localisation. Exp Cell Res. 256: 522-532.

PARKER, F., MAURIER, F., DELUMEAU, I., DUCHESNE, M., FAUCHER, D. DEBUSSCHE, L., DUGUE, A., SCHWEIGHOFFER, F. and TOCQUE, B. (1996). A ras-gtpase-activating protein sh3-domain-binding protein. Mol. Cell Biol. 16: 2561-2569.

PAZMAN, C., MAYES, C., FANTO, M., HAYNES, S. and MLODZIK, M. (2000). Rasputin, the drosophila homologue of the rasgap sh3 binding protein functions in ras- and rho-mediated signaling. Development 127: 1715-25.

POMERANCE, M., THANG, M., TOCQUE, B. and PIERRE, M. (1996). The rasgtpase-activating protein sh3 domain is required for cdc2 ctivation and mos induction by oncogenic as in xenopus oocytes independently of mitogenactivated protein kinase activation. Mol. Cell Biol. 16: 3179-3186.

PRIGENT, M., BARLAT, I., LANGEN, H. and DARGEMONT, C. (2000). Ikba and $\mathrm{ikba} / \mathrm{nfkb}$ complexes are retained in the cytoplasm through interaction with a novel partner, rasgap sh3-binding protein 2. J. Biol. Chem. 275: 36441-9.

RIBBECK, K., LIPOWSKY, G., KENT, H., STEWART, M. and GORLICH, D. (1998). Ntf2 mediates nuclear import of ran. EMBO J17: 6587-6598.

ROSS, J. (1995). Mrna stability in mammalian cells. MicroBiol. Rev.423-50.

SAKSELA, K., CHENG, G. and BALTIMORE, D. (1995). Proline-rich (pxxp) motifs in hiv-1 nef bind to sh3 domains of a subset of src kinases and are required for the enhancement growth of nef+ viruses but not for downregulation of cd4. EMBO J14: 484-91.

SCHMITZ, A.A., GOVEK, E.E., BOTTNER, B. and VAN AELST, L. (2000). Rho gtpases: Signaling, migration and invasion. Exp Cell Res 261: 1-12.

SCHNELL, J. and HICKE, L. (2003). Non-traditional functions of ubiquitin and ubiquitin-binding proteins. J Biol Chem 278: 35857-35860

SIMON, M., BOWTELL, D., DODSON, G., LAVERTY, T. and RUBIN, G. (1991) Ras1 and putative guanine nucleotide exchange factor perform crucial steps in the signaling by the sevenless protein tyrosine kinase. Cel/67: 701-716.

SMITH, A., BROWNAWELL, A. and MACARA, I. (1998). Nuclear import of ran is mediated by the transport factor ntf2. Curr. Biol. 25: 1403-6.

SONCINI, C., BERDO, I. and DRAETTA, G. (2001). Ras-gap sh3 domain binding protein (g3bp) is a modulator of usp10-, a novel human ubiquitin specific protease. Oncogene 20: 3869-3879.

SONENBERG, N. and GINGRAS, A. (1998). The mrna 5' cap-binding protein eif4e and control of cell growth. Curr. Op. Cell Biol. 10: 268-275.

STOECKLIN, G., GROSS, B., MING, X.F. and MORONI, C. (2003). A nove mechanism of tumor suppression by destabilizing au-rich growth factor mrna. Oncogene 22: 3554-61.

STRUTT, D. (2003). Frizzled signalling and cell polarisation in drosophila and vertebrates. Development 130: 4501-13.

SUEOKA, E., GOTO, Y., SUEOKA, N., KAI, Y., KOZU, T. and FUJIKI, H. (1999). Heterogeneous nuclear ribonucleoprotein b1 as a new marker of early detection for human lung cancers. Cancer Res 59: 1404-7.

TOCQUE, B., DELUMEAU, I., PARKER, F., MAURIER, F., MULTON, M. and SCHWEIGHOFFER, F. (1997). Ras-gtpase activating protein (gap): A putative effector for ras. Cell Signaling 9: 153-158.

TOURRIERE, H., CHEBLI, K., ZEKRI, L., COURSELAUD, B., BLANCHARD, J.M., BERTRAND, E. and TAZI, J. (2003). The rasgap-associated endoribonuclease g3bp assembles stress granules. J Cell Bio/160: 823-31.

TOURRIERE, H., GALLOUZI, I., CHEBLI, K., CAPONY, J., MOUAIKEL, J., VAN DER GEER, P. and TAZI, J. (2001). Rasgap-associated endoribonuclease 
g3bp; selective rna degradation and phosphorylation-dependent localisation. Mol. Cell Biol. 21: 7747-7760.

WHITESIDE, S. and ISRAEL, A. (1997). Ikappab proteins: Structure function and regulation. Cancer Biol. 8: 75-82.

WHITFIELD, M., SHERLOCK, G., SALDANHZ, A., MURRAY, J., BALL, C., ALEXANDER, K., MATESE, J., PEROU, C., HURT, M., BROWN, P. et al. (2002). Identification of genes periodically expressed in the human cell cycle and their expression in tumours. Mol Biol Cel/13: 1977-2000.

WILKINSON, K. (2003). Aspirin, ubiquitin and cancer. Nature 424: 738.
YARDEN, Y. (2001). Biology of her2 and its importance in breast cancer. Oncology 61: 1-13.

YEUNG, Y., WANG, Y., EINSTEIN, D., LEE, P. and STANLEY, E. (1998). Colonystimulating factor-1 stimulates the formation of multimeric cytosolic complexes of signaling proteins and cytoskeletal components in macrophages. $J$ Biol Chem 273: 17128-12137.

Received: June 2004

Reviewed by Referees: July 2004

Modified by Authors and Accepted for Publication: August 2004 Edited by: Patrick Tam 